\title{
Timing of AKI after urgent percutaneous coronary intervention and clinical outcomes: a high-dimensional propensity score analysis
}

Alan S. Go ${ }^{1,2,3,4^{*}}$, Thida C. Tan ${ }^{1}$, Rishi V. Parikh ${ }^{1}$, Andrew P. Ambrosy ${ }^{1,5}$, Leonid V. Pravoverov ${ }^{6}$, Sijie Zheng ${ }^{1,6,7}$ and Thomas K. Leong ${ }^{1}$

\begin{abstract}
Introduction: Acute kidney injury is a common complication of percutaneous coronary intervention and has been associated with an increased risk of death and progressive chronic kidney disease. However, whether the timing of acute kidney injury after urgent percutaneous coronary intervention could be used to improve patient risk stratification is not known.

Methods: We conducted a retrospective cohort study in adults surviving an urgent percutaneous coronary intervention between 2008 and 2013 within Kaiser Permanente Northern California, a large integrated healthcare delivery system, to evaluate the impact of acute kidney injury during hospitalization at $12( \pm 6), 24( \pm 6)$ and $48( \pm 6)$ hours after urgent percutaneous coronary intervention and subsequent risks of adverse outcomes within the first year after discharge. We used multivariable Cox proportional hazards models with adjustment for a highdimensional propensity score for developing acute kidney injury after percutaneous coronary intervention to examine the associations between acute kidney injury timing and all-cause death and worsening chronic kidney disease.

Results: Among 7250 eligible adults undergoing urgent percutaneous coronary intervention, 306 (4.2\%) had acute kidney injury at one or more of the examined time periods after percutaneous coronary intervention. After adjustment, acute kidney injury at $12( \pm 6)$ hours was independently associated with higher risks of death (adjusted hazard ratio [aHR] 3.55, 95\% confidence interval [Cl] 2.19-5.75) and worsening kidney function (aHR 2.40, 95\% Cl: 1.24-4.63). Similar results were observed for acute kidney injury at $24( \pm 6)$ hours and death (aHR 3.90, 95\% Cl:2.29$6.66)$ and worsening chronic kidney disease (aHR 4.77, 95\% Cl:2.46-9.23). Acute kidney injury at 48 ( \pm 6 ) hours was associated with excess mortality (aHR 1.97, 95\% Cl:1.19-3.26) but was not significantly associated with worsening kidney function (aHR 0.91, 95\% Cl:0.42-1.98).
\end{abstract}

\footnotetext{
* Correspondence: Alan.S.Go@kp.org

'Division of Research, Kaiser Permanente Northern California, 2000 Broadway, Oakland, CA 94549, USA

${ }^{2}$ Department of Health Systems Science, Kaiser Permanente Bernard J. Tyson School of Medicine, Pasadena, CA, USA

Full list of author information is available at the end of the article
}

(c) The Author(s). 2021 Open Access This article is licensed under a Creative Commons Attribution 4.0 International License, which permits use, sharing, adaptation, distribution and reproduction in any medium or format, as long as you give appropriate credit to the original author(s) and the source, provide a link to the Creative Commons licence, and indicate if changes were made. The images or other third party material in this article are included in the article's Creative Commons licence, unless indicated otherwise in a credit line to the material. If material is not included in the article's Creative Commons licence and your intended use is not permitted by statutory regulation or exceeds the permitted use, you will need to obtain permission directly from the copyright holder. To view a copy of this licence, visit http://creativecommons.org/licenses/by/4.0/. The Creative Commons Public Domain Dedication waiver (http://creativecommons.org/publicdomain/zero/1.0/) applies to the data made available in this article, unless otherwise stated in a credit line to the data. 
Conclusions: Timing of acute kidney injury after urgent percutaneous coronary intervention may be differentially associated with subsequent risk of worsening kidney function but not death.

Keywords: Acute kidney injury, Percutaneous coronary intervention, Timing, Chronic kidney disease, Death

\section{Introduction}

Percutaneous coronary intervention (PCI) remains a first-line therapy for patients with acute coronary syndromes across the spectrum of pre-existing kidney function [1]. Acute kidney injury (AKI) is a well-recognized complication of PCI, $[2,3]$ with previous studies showing that AKI occurring after PCI is associated with adverse events, including incident and progressive chronic kidney disease (CKD) and death [4-8].

While there are various prior studies of the impact of AKI after PCI on future clinical outcomes with a particular focus on intravenous contrast-related management, more accurate identification of high-risk patients is needed to improve cost-effective follow-up and secondary prevention strategies, [9] especially in the current COVID-19 pandemic that has shifted a significant proportion of cardiovascular care to remote approaches [10]. At present, there is a knowledge gap in the field about when and whether to intervene peri-PCI to try to prevent AKI, as well as uncertainty about optimal preventive or management approaches related to AKI. Importantly, it remains unclear whether early detection of AKI (e.g., within 12 to $24 \mathrm{~h}$ ) after PCI could be used to improve patient risk stratification or inform provider decisions to try to reduce the risk of subsequent AKI-related outcomes. Further complicating this is that the definition of AKI used postPCI varies widely across studies and has not been restricted to increases in serum creatinine measurements that occur earlier than $48 \mathrm{~h}$ after PCI [11-13]. Additionally, the development of AKI after PCI is known to have multiple risk factors and possible mechanisms, [14-17] and it is unclear if episodes of AKI occurring at different times after PCI are related to different causes and contribute variably to different natural histories and implications for future clinical outcomes.

Our study focused on addressing the knowledge gap about whether timing of $\mathrm{AKI}$ in patients undergoing urgent PCI was independently and differentially associated with mortality and CKD progression after hospital discharge. We hypothesized that patients who developed AKI at different time points after PCI would experience variable subsequent risks of death and reduced kidney function than patients who do not develop AKI.

\section{Methods}

\section{Source population}

Kaiser Permanente Northern California (KPNC) is a large integrated health care delivery system currently providing comprehensive outpatient, emergency department and inpatient care to $>4.5$ million persons in northern and central California. The KPNC membership is highly representative of the local surrounding and statewide population in terms of age, gender, race/ethnicity and socioeconomic status [18]. Nearly all aspects of care are captured through an integrated electronic health record system, with key variables extracted and standardized for research in the Kaiser Permanente Virtual Data Warehouse (VDW) [19].

This study was approved by the Kaiser Permanente Northern California institutional review board. We obtained a waiver of informed consent from the Kaiser Permanente Northern California institutional review board as the risk to patients was considered minimal given the nature of this retrospective data-only study. All methods were carried out in accordance with relevant guidelines and regulations.

\section{Study eligibility}

We initially identified all adult ( $\geq 18$ years) patients who received a PCI between January 1, 2008 and December 31, 2013 in KPNC facilities. Using previously validated methods based on electronic health records and billing claims data [20], we defined PCI using International Classification of Diseases, Version 9 (ICD-9) or Current Procedural Terminology (CPT) procedure codes. Furthermore, we identified a subset of all adults receiving PCI who had detailed information on their procedure available in a regional cardiac catheterization laboratory data repository. We excluded patients who did not have an outpatient estimated glomerular filtration rate (eGFR) measurement within 12 months before the PCI, or who had Stage 5 CKD at the time of PCI based on Kidney Disease Outcomes Quality Initiative criteria [21]. We also excluded patients with less than 12 months of continuous health plan membership before their PCI or having a history of cirrhosis, organ transplantation, or renal replacement therapy. Finally, given we were interested in AKI in the high-risk setting of urgent PCI, we only included patients receiving a $\mathrm{PCI}$ as part of an acute inpatient or emergency department encounter for acute coronary syndrome. Each patient's first qualifying urgent PCI during the study period was assigned as their index date.

\section{Acute kidney injury}

Our predictor variable was AKI occurring at $12( \pm 6)$ hours, $24( \pm 6)$ hours and $48( \pm 6)$ hours post-PCI during 
the index hospitalization. We defined AKI as a $\geq 50 \%$ relative increase or $\mathrm{a} \geq 0.3 \mathrm{mg} / \mathrm{dL}$ absolute increase in serum creatinine relative to the most recent pre-PCI outpatient serum creatinine measure, based on the Kidney Disease: Improving Global Outcomes (KDIGO) criteria [22]. We also characterized each instance of AKI as either Stage 1 AKI or Stage 2/3 AKI based on KDIGO serum creatinine criteria as urine output data were unavailable. We assessed AKI status for each time period independently, such that each patient was allowed to be considered to have AKI in multiple time periods. For each analysis of timingspecific AKI, we excluded any patients who did not have a serum creatinine measurement in the prespecified time window post-PCI.

\section{Follow-up and outcomes}

Patients were followed from hospital discharge to the first occurrence of any of the following events: death, disenrollment from the health plan, non-renal organ transplantation or end of the study follow-up period on December 31, 2014.

Our primary outcome was death from any cause, based on a previously validated comprehensive approach using electronic health records, health plan administrative data (including proxy reporting), Social Security vital status information and state death certificate data [23].

For the subgroup of patients with CKD before the $\mathrm{PCI}$, we also examined post-discharge significant loss of kidney function, which we defined as a $50 \%$ relative decrease from pre-PCI eGFR using the CKD-EPI equation [24] or development of end-stage kidney disease (ESKD) defined as receipt of kidney replacement therapy based on a comprehensive health plan ESKD registry [25].

\section{Covariates}

We identified patients with a history of proteinuria, defined as a urinary albumin/creatinine ratio $\geq 30 \mu \mathrm{g} /$ $\mathrm{mg}$, a urinary protein/creatinine ratio $\geq 0.3 \mathrm{mg} / \mathrm{mg}$ or a 24-h urinary protein measurement $\geq 150 \mathrm{mg}$ up to 4 years before the index urgent PCI using health plan laboratory results data. We used the most recent prePCI serum creatinine measure to identify patients with pre-existing stage 3 or 4 CKD. We also collected all available inpatient and outpatient diagnosis codes, procedure codes and pharmacy dispensing records data up to 4 years prior to PCI for use in our highdimensional propensity score estimation. Additionally, we identified clinically relevant comorbidities up to 4 years prior to $\mathrm{PCI}$ using previously validated diagnosis codes, procedure codes, laboratory and prescription medication dispensing data [26].

\section{Statistical approach}

We used SAS statistical software, version 9.3 (Cary, NC) for all analyses, with a two-sided $P<0.05$ being considered significant. We compared baseline characteristics across AKI status at each time point using analysis of variance for continuous variables and chi-square tests for categorical variables.

To adjust for potential unmeasured confounding, we estimated high-dimensional propensity scores (hd-PS) [27] for AKI at $12( \pm 6), 24( \pm 6)$ and $48( \pm 6)$ hours postPCI, as well as for AKI at any of the three time points. To generate the hd-PS, we performed multivariable logistic regression for predicting development of AKI at each time period post-PCI using pre-PCI patient demographics, diagnoses, procedures and prescription medications, with variables selected by an algorithm that identified and prioritized candidate variables based on the empirical association between the candidate variable and the event $[28,29]$. This methodology has been shown to approximate estimates of risk from randomized trials substantially better than standard propensity scoring or regression approaches [27, 30]. The final hdPS models each included 150 algorithmically selected variables and showed excellent model discrimination for AKI at $12( \pm 6)$ hours (c-statistic 0.83$)$, at $24( \pm 6)$ hours (c-statistic 0.90$)$ and at $48( \pm 6)$ hours (c-statistic 0.89$)$ post-PCI.

We conducted Cox proportional hazards models to assess the association between AKI at different time points post-PCI and each outcome of interest at 1 year of follow-up, with adjustment for pre-PCI eGFR, proteinuria status and the hd-PS for AKI at the appropriate time period. To address the possibility that AKI severity may differ across the timepoints assessed, we also conducted sensitivity analyses of the association between Stage 2/3 AKI post-PCI and all-cause mortality as well as CKD progression. We also conducted proportional hazards models for post-discharge loss of kidney function using the Fine-Gray subdistribution approach to account for death as a competing risk as an additional sensitivity analysis. Furthermore, to examine whether cardiogenic shock could account for an association between AKI and death, we conducted a sensitivity analysis excluding patients who received a diagnosis of cardiogenic shock during the index hospitalization.

\section{Results}

Study cohort and baseline characteristics by AKI status

Among the 7250 eligible patients undergoing urgent PCI identified, mean age was 67 years, $29 \%$ were women, $5 \%$ were Black and $16 \%$ were Asian/Pacific Islander (Fig. 1). Among these patients, we identified 5484 eligible patients with serum creatinine measurements at $12( \pm 6)$ hours, 2132 eligible patients with serum creatinine 
measurements at $24( \pm 6)$ hours, and 906 eligible patients with serum creatinine measurements at $48( \pm 6)$ hours post-PCI during hospitalization (Fig. 1). Of note, 2242 $(40.9 \%)$ in the $12( \pm 6)$ hours cohort, $979(45.9 \%)$ in the $24( \pm 6)$ hours cohort and $477(52.6 \%)$ in the $48( \pm 6)$ hours cohort had prior Stage 3/4 CKD or proteinuria.

Across all time periods, we identified AKI in a total of 306 patients, with 152 (3\%) of those with serum creatinine measured at $12( \pm 6)$ hours, $140(7 \%)$ of those measured at $24( \pm 6)$ hours, and $147(16 \%)$ of those measured at $48( \pm 6)$ hours. Among the identified AKI episodes, $93 \%$ of those measured at $12( \pm 6)$ hours, $85 \%$ of those measured at $24( \pm 6)$ hours and $71 \%$ of those measured at $48( \pm 6)$ hours were classified as Stage 1 AKI. At the individual patient level, 108 patients (35\%) had AKI at more than one of the pre-specified time points post-PCI, with 25 (8\%) having AKI at all three time points.

Patients with documented AKI at any time point were more likely than those without AKI to be older and women, and to have a lower eGFR pre-PCI (Table 1). In addition, patients with AKI were more likely to have a prior history of proteinuria, hypertension, dyslipidemia, diabetes mellitus and heart failure.

\section{Follow-up and outcomes}

Mean (SD) follow-up after hospital discharge was 344 (72) days, with 403 (5.5\%) observed deaths within the first year post-discharge. In addition, among 3020 patients with CKD pre-PCI, 125 (4.1\%) patients experienced progressively worsening CKD during the first year of follow-up post-discharge.

\section{Timing of AKI and outcomes}

In multivariable Cox proportional hazards models, compared to patients who did not experience AKI, patients who experienced AKI at any examined time point postPCI had an increased rate of all-cause death at 1-year post-discharge, with an adjusted hazard ratio (aHR) of 3.09 (95\% confidence interval [CI] 2.19-4.36). In patients with serum creatinine measurements at $12( \pm 6)$ hours post-PCI, those experiencing AKI had a more than 3.5fold higher adjusted rate of all-cause death (aHR 3.55, 95\% CI:2.19-5.75) compared to patients without AKI. Similarly, among those with serum creatinine measurements at $24( \pm 6)$ hours post-PCI, those with AKI had a nearly fourfold higher adjusted rate of death (aHR 3.90, 95\% CI:2.29-6.66). In patients with serum creatinine

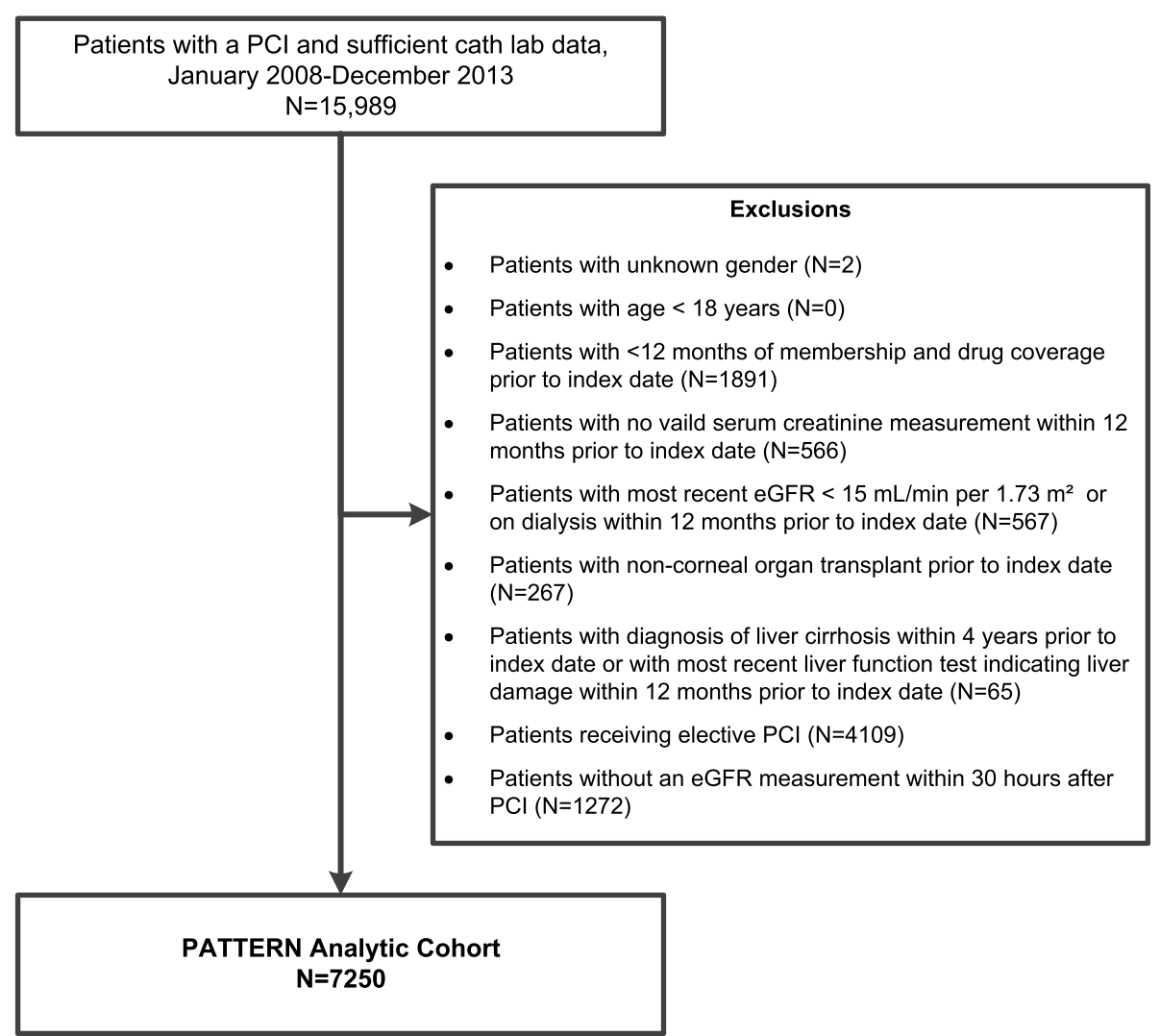

Fig. 1 Cohort assembly diagram of adults with and without post-procedural AKI after urgent percutaneous coronary intervention identified between January 2008 through December 2013 
Table 1 Baseline characteristics of patients with and without post-procedural AKI after urgent percutaneous coronary intervention identified between January 2008 - December 2013

\begin{tabular}{|c|c|c|c|c|}
\hline & & $48 \mathrm{~h}$ post- $\mathrm{P}$ & & \\
\hline & Overall & No AKI & AKI & D-value \\
\hline & $(N=7250)$ & $(N=6944)$ & $(N=306)$ & \\
\hline \multicolumn{5}{|l|}{ Sociodemographic characteristics } \\
\hline Mean (SD) age, yr & $67(12)$ & $67(12)$ & $72(12)$ & 0.47 \\
\hline Women, No. (\%) & $2074(29)$ & $1949(28)$ & $125(41)$ & 0.27 \\
\hline \multicolumn{5}{|l|}{ Race, No. (\%) } \\
\hline White & $4472(62)$ & $4300(62)$ & $172(56)$ & 0.23 \\
\hline Black/African American & $378(5)$ & $358(5)$ & $20(67)$ & \\
\hline Asian/Pacific Islander & $1146(16)$ & $1108(16)$ & $38(12)$ & \\
\hline Other/Unknown & $1254(17)$ & $1178(17)$ & $76(25)$ & \\
\hline Hispanic ethnicity, No. (\%) & $921(13)$ & $865(12)$ & $56(18)$ & 0.16 \\
\hline Index PCl event, No. (\%) & & & & 0.24 \\
\hline No incident Ml & $1280(18)$ & $1248(18)$ & $32(11)$ & \\
\hline NSTEMI & $4486(62)$ & $4283(62)$ & $203(66)$ & \\
\hline STEMI & $1484(20)$ & $1413(20)$ & $71(23)$ & \\
\hline \multicolumn{5}{|l|}{ Medical history, No. (\%) } \\
\hline Acute myocardial infarction & $501(7)$ & $471(7)$ & $30(10)$ & 0.11 \\
\hline Unstable angina & $77(1)$ & $74(1)$ & $3(1)$ & 0.01 \\
\hline Coronary artery bypass graft & $118(1)$ & $117(1)$ & $1(0)$ & 0.14 \\
\hline Percutaneous coronary intervention & $574(8)$ & $553(8)$ & $21(7)$ & 0.04 \\
\hline Atrial fibrillation or flutter & $581(8)$ & $543(8)$ & $38(12)$ & 0.15 \\
\hline Ischemic stroke/transient ischemic attack & $235(3)$ & $218(3)$ & $17(6)$ & 0.12 \\
\hline Heart failure & $729(10)$ & $665(10)$ & $64(21)$ & 0.32 \\
\hline Peripheral artery disease & $205(3)$ & $180(3)$ & $25(8)$ & 0.25 \\
\hline Hypertension & $5182(72)$ & $4905(71)$ & $277(91)$ & 0.52 \\
\hline Dyslipidemia & $5520(76)$ & $5250(76)$ & $270(88)$ & 0.33 \\
\hline Diabetes mellitus & $2397(33)$ & $2221(32)$ & $176(58)$ & 0.53 \\
\hline Hypothyroidism & $848(12)$ & $794(11)$ & $54(18)$ & 0.18 \\
\hline Chronic lung disease & $1848(26)$ & $1769(26)$ & $79(26)$ & 0.01 \\
\hline Diagnosed dementia & $171(2)$ & $163(2)$ & $8(3)$ & 0.02 \\
\hline Diagnosed depression & $1003(14)$ & $942(14)$ & $61(20)$ & 0.17 \\
\hline \multicolumn{5}{|l|}{ Baseline medication, No. (\%) } \\
\hline ACE inhibitor & $2520(35)$ & $2414(35)$ & $106(35)$ & 0.00 \\
\hline Angiotensin II receptor blocker & $1035(14)$ & $963(14)$ & $72(24)$ & 0.25 \\
\hline Beta blocker & $3364(46)$ & $3164(46)$ & $200(65)$ & 0.41 \\
\hline Calcium channel blocker & $1425(20)$ & $1315(19)$ & $110(36)$ & 0.39 \\
\hline Diuretic & $2441(34)$ & $2281(33)$ & $160(52)$ & 0.40 \\
\hline Aldosterone receptor antagonist & $99(1)$ & $93(1)$ & $6(2)$ & 0.05 \\
\hline Alpha blocker & $772(11)$ & $712(10)$ & $60(20)$ & 0.26 \\
\hline Statin & $3980(55)$ & $3781(54)$ & $199(65)$ & 0.22 \\
\hline Other lipid-lowering agent & $478(7)$ & $454(7)$ & $24(8)$ & 0.05 \\
\hline Non-aspirin antiplatelet agent & $713(10)$ & $657(10)$ & $56(18)$ & 0.26 \\
\hline Anticoagulant & $384(5)$ & $362(5)$ & $22(7)$ & 0.08 \\
\hline
\end{tabular}


Table 1 Baseline characteristics of patients with and without post-procedural AKI after urgent percutaneous coronary intervention identified between January 2008 - December 2013 (Continued)

\begin{tabular}{|c|c|c|c|c|}
\hline & & $48 \mathrm{~h}$ post & & \\
\hline Diabetic therapy & $1390(19)$ & $1259(18)$ & $131(43)$ & 0.56 \\
\hline \multicolumn{5}{|l|}{ Laboratory results } \\
\hline Qualifying estimated glomerular filtration rate, $\mathrm{ml} / \mathrm{min} / 1.73 \mathrm{~m}^{2}$, No. (\%) & & & & 0.92 \\
\hline$>150$ & $1(0)$ & $1(0)$ & $0(0)$ & \\
\hline $90-150$ & $1803(25)$ & $1775(26)$ & $28(9)$ & \\
\hline $60-89$ & $3571(49)$ & $3481(50)$ & $90(29)$ & \\
\hline $45-59$ & $1138(16)$ & $1069(15)$ & $69(23)$ & \\
\hline $30-44$ & $569(8)$ & $492(7)$ & $77(25)$ & \\
\hline $15-29$ & $168(2)$ & $126(2)$ & $42(14)$ & \\
\hline \multicolumn{5}{|l|}{ Documented proteinuria, No. (\%) } \\
\hline Yes & $2036(28)$ & $1850(27)$ & $186(61)$ & 0.73 \\
\hline
\end{tabular}

measurements at $48( \pm 6)$ hours post-PCI, compared to those without AKI, patients experiencing AKI had a twofold higher adjusted rate of death (aHR 1.97, 95\% CI: 1.19-3.26) (Fig. 2A).

Among the subset of patients with CKD prior to PCI, occurrence of AKI at any examined timepoint post-PCI was independently associated with worsening CKD (aHR 2.39, 95\% CI:1.53-3.74). When examining patients at specific timepoints post-PCI, occurrence of AKI at 12 ( \pm 6) hours (aHR 2.40, 95\% CI:1.24-4.63) and at 24 ( \pm 6$)$ hours (aHR 4.77, 95\% CI:2.46-9.23) were both associated with significantly increased adjusted rates of worsening CKD in the first year post-discharge. In contrast, AKI detected at $48( \pm 6)$ hours, was not significantly associated with post-discharge worsening CKD (aHR 0.91, 95\% CI:0.42-1.96) (Fig. 2B).

In sensitivity analyses, more severe (i.e., stage 2 or 3 ) AKI was associated with increased adjusted rates of allcause death at each time point but with a declining strength of association for AKI at later time points: at 12 ( \pm 6$)$ hours (aHR 26.53, 95\% CI:8.51-82.75), at $24( \pm 6)$ hours (aHR 6.01, 95\% CI:2.29-15.77) and at $48( \pm 6)$ hours (aHR 3.52, 95\% CI:1.81-6.85) post-PCI. Similarly, in the subgroup of patients with CKD prior to PCI, more severe AKI was also associated with increased adjusted rates of CKD progression for severe AKI at $12( \pm 6)$ hours (aHR 104.23, 95\% CI: 30.65-354.37), but not at $24( \pm 6)$ hours (aHR 2.34, 95\% CI: $0.45-12.26)$ or at $48( \pm 6)$ hours (aHR 1.04, 95\% CI: 0.36-3.01) post-PCI. Additional sensitivity analyses accounting for competing risk of death produced similar results for the associations of AKI at each time point and subsequent CKD progression.

\section{Sensitivity analysis of AKI timing and death in patients without diagnosed cardiogenic shock}

For the subgroup of patients with no diagnosis of cardiogenic shock during the index hospitalization, in multivariable Cox proportional hazards models, compared to patients who did not experience AKI, patients who experienced AKI at any examined time point postPCI had an increased rate of all-cause death at 1-year post-discharge (aHR 2.15, 95\% CI 1.43-3.22). In patients with serum creatinine measurements at $12( \pm 6)$ hours post-PCI, those experiencing AKI had a more than twofold higher adjusted rate of all-cause death (aHR 2.39, 95\% CI:1.31-4.35) compared to patients without AKI. No significant association was seen between AKI and death among those with serum creatinine measurements at $24( \pm 6)$ hours post-PCI (aHR 1.58, 95\% CI:0.76-3.30) or at $48( \pm 6)$ hours post-PCI (aHR 1.32, 95\% CI:0.702.50).

\section{Conclusions}

Among a diverse cohort of adults undergoing urgent PCI within an integrated healthcare delivery system, we examined the associations between timing of AKI and post-discharge 1-year clinical outcomes. We observed independent associations between early AKI after PCI and all-cause mortality and in the subset of patients with pre-existing CKD, AKI was associated with CKD progression. Of note, in the subgroup of patients without a diagnosis of cardiogenic shock during the index hospitalization, the association of AKI and excess mortality was present at all time points but was only significant for early AKI. The strength and direction of the associations we observed between AKI and the outcomes of death and CKD progression are similar to previously published results for AKI identified at $48 \mathrm{~h}$ or later after PCI. A retrospective study of 453,475 Medicare patients undergoing PCI between 2004 and 2009 found a 2.5-fold increased rate of all-cause death within 1 year for patients with Acute Kidney Injury Network (AKIN) Stage 2/3 AKI compared to patients without AKI detected during the index hospitalization [7]. Similarly, another 


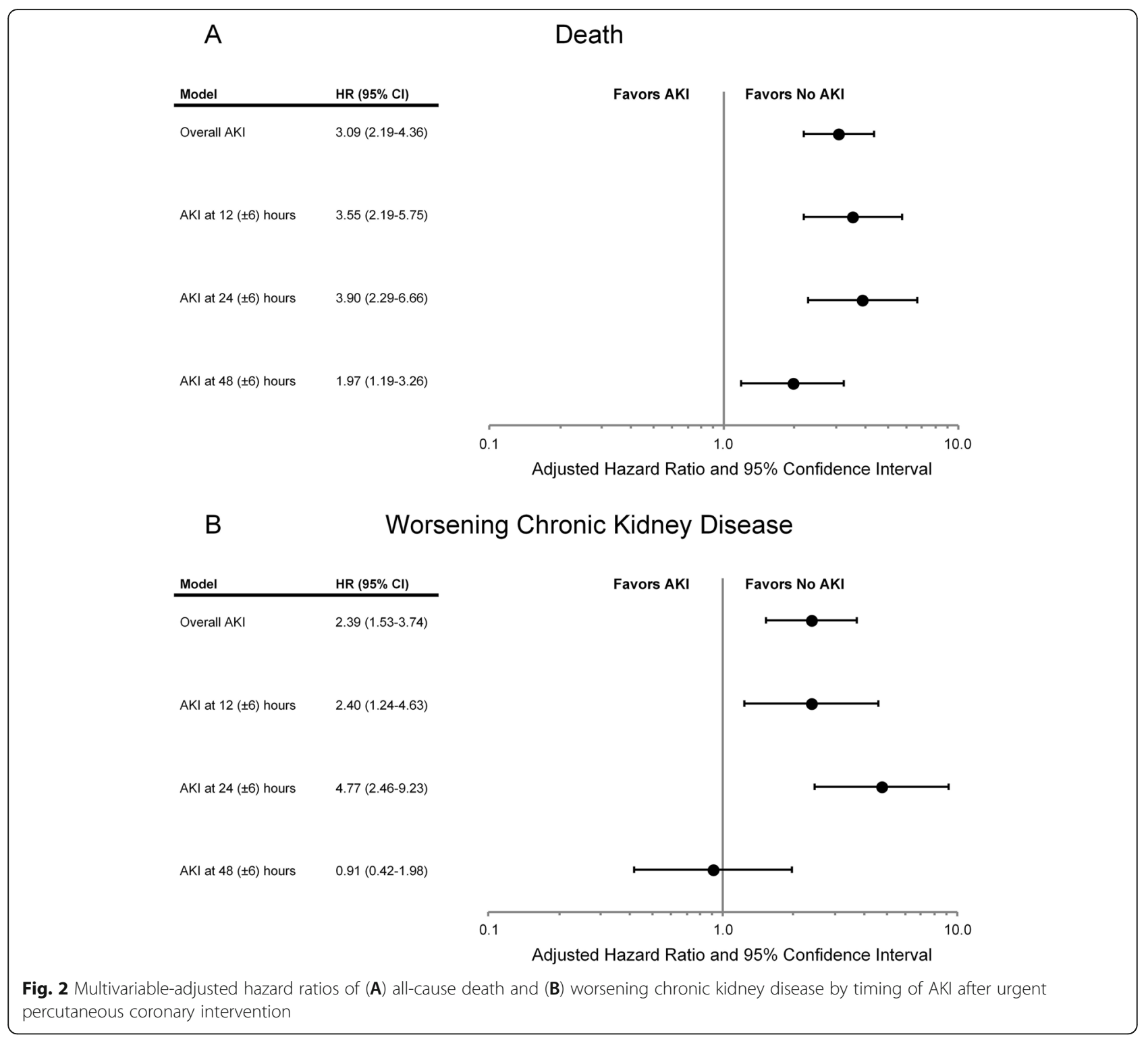

retrospective study of 24,405 patients in the VA Health Care system undergoing PCI between 2005 and 2010 found a twofold higher rate of all-cause death over 5 years for patients developing AKI versus patients without AKI [4]. Our study extends these previous findings by evaluating the development of AKI at three early and specific time periods post-PCI in high risk patients undergoing urgent PCI.

Specifically, AKI identified at both $12( \pm 6)$ and $24( \pm 6)$ hours post-PCI was more strongly associated with death within 1-year post-discharge than AKI identified at $48( \pm$ 6) hours post-PCI. While multiple previous analyses have consistently demonstrated an association between post-PCI AKI and excess all-cause mortality, none of the studies defined AKI based on serum creatinine results obtained less than $48 \mathrm{~h}$ after PCI [8]. Additionally, we found that the relative strengths of associations between AKI at different time points and all-cause death persisted when restricting the analysis to only severe AKI, despite a higher proportion of AKI at $48 \mathrm{~h}$ being considered severe. This suggests that the association of AKI timing with mortality may be mediated through pathways beyond just the severity of the AKI episode. We also found that AKI detected at 12 and $24 \mathrm{~h}$ among those with pre-existing CKD was associated with higher adjusted rates of worsening CKD post-discharge, while AKI at $48 \mathrm{~h}$ was not significantly associated with worsening CKD. These results suggest that AKI developing within $24 \mathrm{~h}$ after urgent PCI may be distinct in terms of subsequent clinical implications.

Our study has several strengths. First, we studied a relatively large cohort of 7250 patients undergoing 
urgent PCI who survived the initial hospitalization, which allowed us to assess the association between AKI episodes at varying times post-PCI using serum creatinine-based criteria and subsequent clinical outcomes within the 1-year post-discharge. Additionally, the comprehensive EHR data available for our cohort enabled calculation of different hd-PS's with excellent discrimination to adjust for potential confounding at each pre-specified post-PCI time point. Our data are also more recent than other previously published studies and included a more contemporary and ethnically diverse population that support greater generalizability of the results.

Our study also had several limitations. As an observational study using serum creatinine data obtained as part of routine clinical care, not every patient received serum creatinine measures at each pre-specified time point. Thus, it is possible that the patients with serum creatinine measurements in earlier vs. later time periods postPCI may be systematically different and also differ from patients without serum creatinine measurements, although a comparison of patients with and without measurements showed that they were similar at baseline. We limited our cohort by design to patients with urgent PCI, so our results may not completely generalize to those undergoing elective PCI in an outpatient setting, although our findings are similar to prior research on AKI occurring at any time involving multiple types of surgery and subsequent outcomes [31]. We also limited our cohort by design to patients with a serum creatinine measurement within 12 months before the index PCI procedures, so it is possible that patients without a recent serum creatinine measurement may be different than those we analyzed in this study. Additionally, we retained patients whose most recent measurement was on the index date, so it is possible that these measurements do not completely reflect the patients' true baseline kidney function. We did not include those with Stage 5 CKD as it is difficult to identify true cases of AKI using serum creatinine-based criteria in such patients. As we did not have any urine output data post$\mathrm{PCI}$, we may have undercounted the incidence of AKI if patients did not qualify for analysis by serum creatinine criteria. We focused on all-cause mortality as a primary endpoint and CKD progression as a secondary endpoint to be most comparable with previous studies and did not address potential variable associations with cardiovascular outcomes or cardiac-related mortality, as we lacked systematic cause of death data for the cohort. We also did not have data on prehydration prior to PCI or on any IV fluids received during PCI, so were unable to determine the impact of these preventative measures on AKI and subsequent outcomes. The exact reasons for why AKI is linked to worse outcomes after PCI are not fully understood given that patients experiencing AKI may be more likely to have more extensive or complex coronary artery disease, have greater comorbidity burden, suffer hemodynamic instability, or have other characteristics that we could not fully account for in our analyses. In addition, residual kidney damage and worsening CKD after an episode of AKI can contribute to subsequent heart failure and excess all-cause mortality [32]. Our study population was derived from a large integrated healthcare delivery system in California, so our findings may not be fully generalizable to other health systems or geographic areas. Finally, we cannot completely rule out residual confounding in our models, even though we adjusted for a wide range of potential confounders using hd-PS methods, since such methods cannot adjust for unmeasured confounding that does not have a measured proxy.

In conclusion, we found that patients who develop AKI after urgent PCI experienced excess all-cause mortality at 1-year post-discharge, with a stronger association for AKI detected at 12 and $24 \mathrm{~h}$ than for AKI detected at $48 \mathrm{~h}$. Among patients with pre-existing CKD, $\mathrm{AKI}$ at 12 and $24 \mathrm{~h}-$ but not at $48 \mathrm{~h}$-after urgent PCI was also associated with higher adjusted rates of worsening CKD. Accounting for the timing of AKI development may improve risk stratification of patients undergoing urgent PCI. Further research is needed to validate if earlier vs. later AKI post-PCI has a different natural history across the range of clinical complications associated with $\mathrm{AKI}$, and whether potential strategies to prevent or treat AKI vary in effectiveness based on the timing of AKI development.

\section{Abbreviations \\ AKI: acute kidney injury; CKD: chronic kidney disease; CPT: current procedural terminology; eGFR: estimated glomerular filtration rate; ICD: international classification of diseases; KDIGO: Kidney Disease: Improving Global Outcomes; KPNC: Kaiser Permanente Northern California; PCl: percutaneous coronary intervention; VDW: Virtual Data Warehouse}

\section{Acknowledgements \\ None.}

\section{-Funding}

The study was supported by a research grant to Kaiser Permanente Northern California from CSL Behring. Neither the funder or participating institution had any role in study design; collection, analysis, and interpretation of data; writing the report; and the decision to submit the report for publication.

\section{-Availability of data and materials}

The datasets generated and/or analysed during the current study are not publicly available due to requirements by the approving institutional review board, but a fully de-identified dataset may be made available from the corresponding author on reasonable request.

\section{-Authors' contributions}

Research idea and study design: ASG, TCT; data acquisition: ASG, TKL; statistical analysis: ASG, TKL; data analysis/interpretation: ASG, TCT, RVP, APA, LVP, SZ, TKL. Each author contributed important intellectual content during manuscript drafting or revision, accepts personal accountability for the author's own contributions, and agrees to ensure that questions pertaining 
to the accuracy or integrity of any portion of the work are appropriately investigated and resolved. The author(s) read and approved the final manuscript.

\section{Declarations}

\section{-Ethics approval and consent to participate}

The study was approved by the Kaiser Permanente Northern California institutional review board. A waiver of informed consent was obtained from the Kaiser Permanente Northern California institutional review board due to the nature of the study.

\section{-Consent for publication}

Not applicable.

\section{- Competing interests}

Dr. Go reports receiving research funding from the National Institute of Diabetes, Digestive and Kidney Diseases; National Heart, Lung and Blood Institute; National Institute on Aging; and CSL Behring. The other authors have no conflicts of interest to declare.

\section{Author details}

'Division of Research, Kaiser Permanente Northern California, 2000 Broadway, Oakland, CA 94549, USA. ${ }^{2}$ Department of Health Systems Science, Kaiser Permanente Bernard J. Tyson School of Medicine, Pasadena, CA, USA. ${ }^{3}$ Departments of Medicine (Nephrology), Epidemiology and Biostatistics, University of California, San Francisco, CA, USA. ${ }^{4}$ Department of Medicine (Nephrology), Stanford University, Palo Alto, CA, USA. ${ }^{5}$ Department of Cardiology, Kaiser Permanente San Francisco Medical Center, San Francisco, CA, USA. ${ }^{6}$ Department of Nephrology, Kaiser Permanente Oakland Medical Center, Oakland, CA, USA. ${ }^{7}$ Department of Medical Education, University of California, San Francisco, CA, USA.

Received: 26 December 2020 Accepted: 26 August 2021

Published online: 06 September 2021

\section{References}

1. Patel MR, Calhoon JH, Dehmer GJ, Grantham JA, Maddox TM, Maron DJ, et al. ACC/AATS/AHA/ASE/ASNC/SCAI/SCCT/STS 2016 Appropriate Use Criteria for Coronary Revascularization in Patients With Acute Coronary Syndromes: A Report of the American College of Cardiology Appropriate Use Criteria Task Force, American Association for Thoracic Surgery, American Heart Association, American Society of Echocardiography, American Society of Nuclear Cardiology, Society for Cardiovascular Angiography and Interventions, Society of Cardiovascular Computed Tomography, and the Society of Thoracic Surgeons. J Nucl Cardiol. 2017;24(2):439-63.

2. Tsai TT, Patel UD, Chang TI, Kennedy KF, Masoudi FA, Matheny ME, et al. Contemporary incidence, predictors, and outcomes of acute kidney injury in patients undergoing percutaneous coronary interventions: insights from the NCDR Cath-PCI registry. JACC Cardiovasc Interv. 2014;7(1):1-9. https://doi. org/10.1016/j.jcin.2013.06.016.

3. Davenport MS, Perazella MA, Yee J, Dillman JR, Fine D, McDonald RJ, et al. Use of intravenous iodinated contrast Media in Patients with Kidney disease: consensus statements from the American College of Radiology and the National Kidney Foundation. Radiology. 2020;294(3):660-8. https://doi. org/10.1148/radiol.2019192094.

4. Brown JR, Solomon RJ, Robey RB, Plomondon ME, Maddox TM, Marshall EJ, Nichols EL, Matheny ME, Tsai TT, Rumsfeld JS, et al. Chronic Kidney Disease Progression and Cardiovascular Outcomes Following Cardiac Catheterization-A Population-Controlled Study. J Am Heart Assoc. 2016; 5(10)

5. Chawla LS, Amdur RL, Shaw AD, Faselis C, Palant CE, Kimmel PL. Association between AKI and long-term renal and cardiovascular outcomes in United States veterans. Clin J Am Soc Nephrol. 2014;9(3):448-56. https://doi.org/1 0.2215/CJN.02440213.

6. Giacoppo D, Madhavan MV, Baber U, Warren J, Bansilal S, Witzenbichler B, et al. Impact of contrast-induced acute Kidney injury after percutaneous coronary intervention on short- and long-term outcomes: pooled analysis from the HORIZONS-AMI and ACUITY trials. Circ Cardiovasc Interv. 2015;8(8): e002475. https://doi.org/10.1161/CIRCINTERVENTIONS.114.002475.
7. Valle JA, McCoy LA, Maddox TM, Rumsfeld JS, Ho PM, Casserly IP, Nallamothu BK, Roe MT, Tsaei TT, Messenger JC: Longitudinal Risk of Adverse Events in Patients With Acute Kidney Injury After Percutaneous Coronary Intervention: Insights From the National Cardiovascular Data Registry. Circ Cardiovasc Interv 2017, 10(4).

8. Yang Y, George KC, Luo R, Cheng Y, Shang W, Ge S, et al. Contrast-induced acute kidney injury and adverse clinical outcomes risk in acute coronary syndrome patients undergoing percutaneous coronary intervention: a metaanalysis. BMC Nephrol. 2018;19(1):374. https://doi.org/10.1186/s12882-018-11 61-5.

9. Marenzi G, Cosentino N, Bartorelli AL. Acute kidney injury in patients with acute coronary syndromes. Heart. 2015;101(22):1778-85. https://doi.org/1 0.1136/heartinl-2015-307773.

10. American College of Cardiology: COVID-19 Clinical Guidance for the Cardiovascular Team. https://www.acc.org// /media/Non-Clinical/Files-PDFsExcel-MS-Word-etc/2020/02/S20028-ACC-Clinical-Bulletin-Coronavirus.pdf. Accessed September 13, 2020. . In.; 2020.

11. Azzalini L, Spagnoli V, Ly HQ. Contrast-induced nephropathy: from pathophysiology to preventive strategies. Can J Cardiol. 2016;32(2):247-55. https://doi.org/10.1016/j.cjca.2015.05.013.

12. Budano C, Levis M, D'Amico M, Usmiani T, Fava A, Sbarra P, et al. Impact of contrast-induced acute kidney injury definition on clinical outcomes. Am Heart J. 2011;161(5):963-71. https://doi.org/10.1016/j.ahj.2011.02.004.

13. Parsh J, Seth M, Briguori C, Grossman P, Solomon R, Gurm HS. The optimal definition of contrast-induced acute kidney injury for prediction of inpatient mortality in patients undergoing percutaneous coronary interventions. Am Heart J. 2016;175:160-7. https://doi.org/10.1016/j.ahj.2016.02.013.

14. Arbel Y, Fuster V, Baber U, Hamza TH, Siami FS, Farkouh ME. Incidence, determinants and impact of acute kidney injury in patients with diabetes mellitus and multivessel disease undergoing coronary revascularization: results from the FREEDOM trial. Int J Cardiol. 2019;293:197-202. https://doi. org/10.1016/j.ijcard.2019.05.064.

15. Karrowni W, Vora AN, Dai D, Wojdyla D, Dakik H, Rao SV: Blood Transfusion and the Risk of Acute Kidney Injury Among Patients With Acute Coronary Syndrome Undergoing Percutaneous Coronary Intervention. Circ Cardiovasc Interv 2016, 9(9).

16. Shacham Y, Gal-Oz A, Leshem-Rubinow E, Arbel Y, Keren G, Roth A, et al. Admission glucose levels and the risk of acute Kidney injury in nondiabetic ST segment elevation myocardial infarction patients undergoing primary percutaneous coronary intervention. Cardiorenal Med. 2015;5(3):191-8. https://doi.org/10.1159/000430472.

17. Shacham $Y$, Steinvil A, Arbel Y. Acute kidney injury among ST elevation myocardial infarction patients treated by primary percutaneous coronary intervention: a multifactorial entity. J Nephrol. 2016;29(2):169-74. https://doi. org/10.1007/s40620-015-0255-4.

18. NP G. Characteristics of adult health plan members in the northern California region membership, as estimated from the 2011 member health survey. In. Oakland: Kaiser Permanente Medical Care Program; 2013.

19. Go AS, Magid DJ, Wells B, Sung SH, Cassidy-Bushrow AE, Greenlee RT, et al. The cardiovascular research network: a new paradigm for cardiovascular quality and outcomes research. Circ Cardiovasc Qual Outcomes. 2008;1(2): 138-47. https://doi.org/10.1161/CIRCOUTCOMES.108.801654.

20. Kazi DS, Leong TK, Chang TI, Solomon MD, Hlatky MA, Go AS. Association of spontaneous bleeding and myocardial infarction with long-term mortality after percutaneous coronary intervention. J Am Coll Cardiol. 2015;65(14): 1411-20. https://doi.org/10.1016/j.jacc.2015.01.047.

21. National Kidney F. KDOQI clinical practice guidelines for chronic kidney disease: evaluation, classification, and stratification. Am J Kidney Dis. 2002; 39(2 Suppl 1):S1-266.

22. Kidney Disease: Improving Global Outcomes (KDIGO) Acute Kidney Injury Work Group KDIGO Clinical Pracetice Guideline for Acute Kidney Injury. Kidney Int Suppl 2012(2):1-138, Notice, 1, DOl: https:/doi.org/10.1038/kisup.2012.1.

23. Go AS, Lee WY, Yang J, Lo JC, Gurwitz JH. Statin therapy and risks for death and hospitalization in chronic heart failure. JAMA. 2006:296(17):2105-11. https://doi.org/10.1001/jama.296.17.2105.

24. Levey AS, Stevens LA, Schmid CH, Zhang YL, Castro AF 3rd, Feldman HI, et al. A new equation to estimate glomerular filtration rate. Ann Intern Med. 2009;150(9):604-12. https://doi.org/10.7326/0003-4819-150-9-20090505000006.

25. Hsu CY, Chertow GM, McCulloch CE, Fan D, Ordonez JD, Go AS Nonrecovery of kidney function and death after acute on chronic renal 
failure. Clin J Am Soc Nephrol. 2009;4(5):891-8. https://doi.org/10.2215/CJN. 05571008.

26. Lee BJ, Hsu CY, Parikh RV, Leong TK, Tan TC, Walia S, et al. Non-recovery from dialysis-requiring acute kidney injury and short-term mortality and cardiovascular risk: a cohort study. BMC Nephrol. 2018;19(1):134. https://doi. org/10.1186/s12882-018-0924-3.

27. Schneeweiss S, Rassen JA, Glynn RJ, Avorn J, Mogun H, Brookhart MA. Highdimensional propensity score adjustment in studies of treatment effects using health care claims data. Epidemiology. 2009;20(4):512-22. https://doi. org/10.1097/EDE.0b013e3181a663cc.

28. Bansal N, Szpiro A, Reynolds K, Smith DH, Magid DJ, Gurwitz JH, et al. Longterm outcomes associated with implantable cardioverter defibrillator in adults with chronic Kidney disease. JAMA Intern Med. 2018;178(3):390-8, https://doi.org/10.1001/jamainternmed.2017.8462.

29. Rassen JA, Schneeweiss S. Using high-dimensional propensity scores to automate confounding control in a distributed medical product safety surveillance system. Pharmacoepidemiol Drug Saf. 2012;21(Suppl 1):41-9. https://doi.org/10.1002/pds.2328.

30. Garbe E, Kloss S, Suling M, Pigeot I, Schneeweiss S. High-dimensional versus conventional propensity scores in a comparative effectiveness study of coxibs and reduced upper gastrointestinal complications. Eur J Clin Pharmacol. 2013;69(3):549-57. https://doi.org/10.1007/s00228-012-1334-2.

31. Ozrazgat-Baslanti T, Thottakkara P, Huber M, Berg K, Gravenstein N, Tighe P, et al. Acute and chronic Kidney disease and cardiovascular mortality after major surgery. Ann Surg. 2016;264(6):987-96. https://doi.org/10.1097/SLA. 0000000000001582.

32. Ikizler TA, Parikh CR, Himmelfarb J, Chinchilli VM, Liu KD, Coca SG, et al. A prospective cohort study of acute kidney injury and kidney outcomes, cardiovascular events, and death. Kidney Int. 2021;99(2):456-65. https://doi. org/10.1016/j.kint.2020.06.032

\section{Publisher's Note}

Springer Nature remains neutral with regard to jurisdictional claims in published maps and institutional affiliations.

Ready to submit your research? Choose BMC and benefit from:

- fast, convenient online submission

- thorough peer review by experienced researchers in your field

- rapid publication on acceptance

- support for research data, including large and complex data types

- gold Open Access which fosters wider collaboration and increased citations

- maximum visibility for your research: over $100 \mathrm{M}$ website views per year

At $\mathrm{BMC}$, research is always in progress.

Learn more biomedcentral.com/submissions 